\title{
APPLYING CRITICAL DESIGN TO COMMUNICATE CULTURAL INSIGHTS EMPHATICALLY: A CASE STUDY
}

\author{
José DE LA O CAMPOS ${ }^{1,3}$ and David GÜEMES ${ }^{2,3}$ \\ ${ }^{1}$ School of Architecture, Art, and Design, Tecnologico de Monterrey \\ ${ }^{2}$ School of Engineering and Sciences, Tecnologico de Monterrey \\ ${ }^{3}$ Vicerrectoría de Investigación y Transferencia de Tecnología, Tecnologico de Monterrey
}

\begin{abstract}
Keywords: User-centred design, industrial design, speculative design, critical design, critical thinking, Mexico, internet of things, educational innovation
\end{abstract}

\begin{abstract}
This research paper explores Critical Design's use to communicate cultural insights generated through a human-centred design (HCD) research process executed by Industrial Design students. The hypothesis behind this research is that Critical Design could bring an enhanced emphatic reflection on these insights. We deployed this approach to a collaborative project between a major international company that designs and sells ready-to-assemble furniture, kitchen appliances, and home accessories based in Sweden and Tecnologico de Monterrey's School of Architecture, Art, and Design in Mexico City. This design exercise's objective was to understand better the desires, goals, frustrations, and cultural differences of end-users in the field of the connected home in Mexico. The research framework consisted of two stages: i) an explorative stage that involved HCD research activities such as daily studies and co-creation workshops with families, and ii) a creative/reflective stage where the students utilized critical and speculative design tactics to translate cultural insights found through the first stage. This study used a qualitative analysis approach to investigate which method carried more meaningfulness by comparing the students' whitepaper at the end of the first stage and the hypothetical objects' results at the end of the second stage. We found that what we called "Hypothetical Products" can assist an HCD report by taking the insights from the research process, and by using a Critical Design approach, it can bring a more significant dimension on empathy to the findings and data that an abstract written detailed results report.
\end{abstract}

\section{INTRODUCTION}

Human-Centred Design (HCD) research is a creative approach with the principle to "know your user" [1]. This research approach relies on understanding the product's context and users' experiences by moving into the field to observe users and how they interact with any product. HCD research utilizes tactics such as daily studies, cultural probes, and deep-dive interviews to obtain insights about the people it is studying through an emphatic lens [2]; these results are communicated through a document containing a set of design criteria or design principles, design strategies or approaches, competitive analyses, or experience roadmaps [3]. These reports and whitepapers are the sources of evidence of the recorded results; however, some limitations exist on bringing a more emphatic approach and better influencing the report's intended audience. We explore alternative ways of sharing these cultural insights by utilizing design tactics related to Critical and Speculative Design to enhanced emphatic reflection on these insights. To do this comparatively, we were inspired by other design researchers to find meaning from empirical data by holding semi-structured deep-dive interviews with the three company' designers that participated with the students during this exercise.

\section{COMMUNICATING HUMAN-CENTERED DESIGN RESEARCH}

It is well understood the challenges designers find when communicating HCD research results. Designers face sharing ideas with colleagues with different thinking ways, like engineers or marketing, can struggle to bridge the gap between collaborative teams [4]. One way to communicate design 
research is through detailed reports. However, reports linger in danger to become verbose, diminishing people's chance to find time to read them [5]. Alternatively, Segelström argues that visual representation is useful among designers who wish to formulate insights from the user material collected and communicate insights to their clients and keep the data "alive" [6]. However, generating these visual representations can have limitations to understand people in an empathic way. A popular visualization tool like a Customer Journey Map can help understand an organization's customer experience. However, there are instances when it is not done correctly; in that case, it can just function as a graphical representation of a customer's touchpoints with an organization, leaving behind all the emotional aspects of the experience, resulting in a useless tool to promote innovation within a service system [7]. Another visualization tool described by Segelström is the Personas Method, which works as a representation of ideal or prototypical end-user based on behaviours and motivations. This method allows designers to relate to and empathize with users and encourage them to view product problems from the user's perspective [8]. As Personas is a subjective representation, it is challenging to verify that the method is accurate as it is complicated to validate, making it useful only for inspiration where there is no claim for validity [9]. As one of the main goals for HCD research is to bring as much information as possible to bring a better understanding of a particular segment of users; the designer must use a plethora of methods and techniques that could complement each other and bring a complete picture, especially when a detailed report, journey maps or personas can be limiting from the empathic point of view.

In the next section, we will open a case for Critical Design as a tactic to improve the empathic dimension for HCD research.

\section{THE HYPOTHETICAL PRODUCT}

Traditional design practice is highly rhetorical, where the designed object is persuasive when it creates a new vision in social life by communicating usability, usefulness, and desirability. Nevertheless, design can also seek an intellectual outcome [10]. Such design practice can be Critical Design. According to Malpass, Critical Design is used as a medium to engage user audiences and provoke debate. With other converging design manifestations such as Design Fiction or Speculative Design, Critical Design relies on hypothetical or fictional objects to explore possible technological futures. Through debate and interaction with these hypothetical products, an audience can consider matters more immediately than abstract theories [11]. A clear example of these hypothetical products is what Kirby famously refers to as diegetic prototypes, a prop-like artifact that, within narratives, contextualizes emergent technologies within the social sphere [12]. Hypothetical products can be used as physical anecdotes designed to prompt speculation in the viewer about the world these objects belong to [13]. A particular characteristic of these hypothetical products (and man-made products in general) is the symbolic qualities in the context of their use, where not only the physical and physiological functions are considered, but the psychological, social, and cultural context. A product can carry a message using a sort of vocabulary of forms that can transfer informative messages that can report possible uses and cultural heritage, individual experiences, and style. By engaging product design, one can be cast in the role of communicator, using the psychological and social uses of objects through the symbolic qualities of products, as man-made forms can embody, like an indistinguishable symbolic unity, both signs and referents relative to a community of users [13]. In this case, this academic exercise appealed to hypothetical functional objects, or as we will call in this paper, hypothetical products, to create this urgency to reckon concerns on the Mexican culture that a user research report might not communicate in the same engaging ways as a product could. This paper explores how a hypothetical object might give a bigger perspective than an abstract insight.

\section{APPLYING CRITICAL DESIGN TO COMMUNICATE CULTURAL INSIGHTS EMPATHICALLY: A CASE STUDY}

A Swedish international company that designs and sells ready-to-assemble furniture, kitchen appliances, and home accessories wanted a deeper understanding of their future Mexican customers. The company paired with Tecnologico de Monterrey's School of Architecture, Art, and Design in Mexico City. For this challenge, undergraduate industrial design students applied User-Centred, Critical, and Speculative Design to gather information about how Mexican families related to smart home devices. The project had two main stages. The first one focused on HCD Research. After doing secondary research and constructing a general scope of the research question, every team recruited a Mexican family and lent 
them a collection of smart products manufactured by the company for a week. During this period, the students gathered insights through HCD activities like a daily study and a co-creating workshop. In the second stage, focused on Critical Design, each team developed a hypothetical collection of smart devices that expressed the Mexican user's emotional needs, aspirations, and frustrations found by their research (Figure 1.1). This project's results were presented in October 2020, during a major design festival in Mexico City. It is worth mention that this semester-long project was temporarily interrupted by the Covid-19 pandemic in its second phase. As the students did not have access to the university facilities, the main deliverables were digital renders and other visualizations. Having described the project and the method, the next part of this paper presents four cases and the analysis.

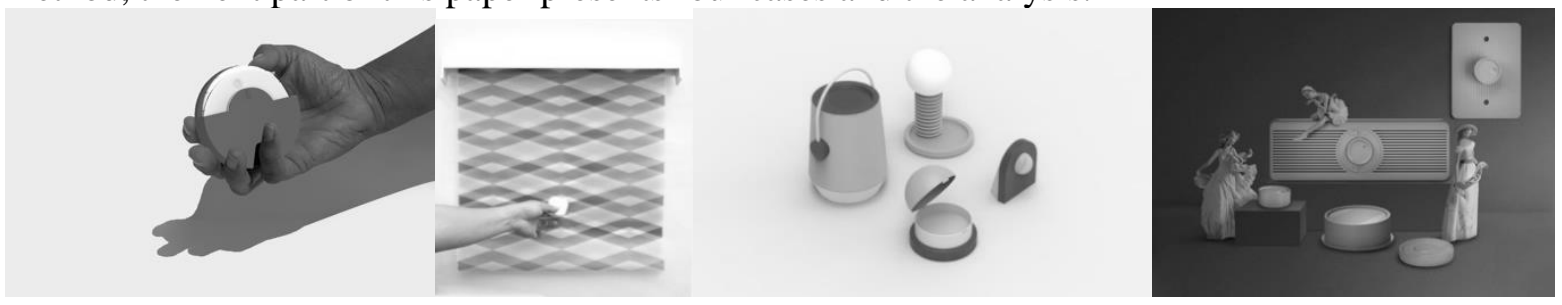

Figure 1. Hypothetical Products developed by the students

\subsection{Team1: Easy}

Through HCD research, the first team found that older Mexican adults (aged 50 to 60) can feel "technophobic" while using smart home devices. After carefully observing how older users felt intimidated by technology, the team found that they perceived "mistrust" and "overwhelm" while exposed to smart devices, causing a dependency on the family's younger members. The students concluded that Mexican users might not be as familiar with smart devices as their European counterparts, making the current design language not universal. As a critical design approach, the team investigated what this type of user would be familiar with in terms of technological devices. They discovered these users would trust more tangible interactions: buttons, sliders, and switches; they also found inspiration on electronics from the ' $70 \mathrm{~s}$ and ' 80 s, where buttons would do loud clicks. After this, they researched ways an older user could feel more familiar with the technology. Therefore, the team created a collection of interactive possibilities aiming to challenge the conception that technological objects should be clean, minimal, and predominantly using white finishes.

\subsection{Team 2: Negotiation of the Space}

The second team of students dived into how Mexican families lived in smaller places with constant space negotiations. During the first stage, the team found that this family had two sisters sharing the same room and the same bed. The older sister, a university student, had a completely different schedule as her younger sister, a high school student. For them, it was challenging to do various activities while sharing the same limited space. In the second stage of the project, this team observed that it was very common to use textiles to separate spaces as a substitute for doors. This team proposed changing the existing smart blind's textile patterns used during the daily study as they found that the Mexican user would be more comfortable when there are more colours and visual elements in their surroundings. Inspired by folk art, this team rethought the smart blind for a Mexican context.

\subsection{Team 3: Lumetría}

The third team dived into the emotional needs the Mexican society has towards celebrating traditions. After interviewing and observing their corresponding family, they saw their meaningful relationship with the social gatherings. As they found out, Mexican families prefer to gather socially in their own homes rather than public spaces. This team focused on how smart lighting could create different environments for different moods and celebrations. A Christmas atmosphere is entirely different from a "Day of the Dead" celebration. This team designed a collection of smart luminaries that could create different atmospheres according to the celebrations. The students turned to the posthumous work of Mexican architect Luis Barragán and used light and colour as an inspiration. The team proposed a collection of three lighting devices: one was fixed in the walls, and two tabletop lamps created colour and light combinations that could generate different home atmospheres. 


\subsection{Team 4: Remembrance Collection}

Finally, during their first stage, the fourth team found opportunities in how older people relate to smart devices. After analysing how their studied family used the smart home devices, they found the grandmother perceived these devices as delicate and fragile - even though they were not. She wanted to feel confident and included while using these devices. Inspired by these insights, the team proposed an alternative design of the company's smart home devices by changing the physical appearance to arouse the user's predominant characteristics that they could remember from their youth. This finding concerned the team, as one of the students explains: "Everyone should be able to use a smart device no matter their age. So we created a collection that, through its appearance, could invite our grandparents to use these home smart devices." By looking into how the design looked before the digital era and using visual cues that could remind us of art-deco details popularized in Mexican households in the '60s, this team of students challenged the paradigm that technology should look like something futuristic, technocratic, or new. At the end of the project, a white paper and project documentation was delivered to the companies' designer, and a final presentation with different stakeholders was organized to share their results.

\section{METHODOLOGY}

To understand the effectiveness of how Critical Design can assist a User Research Report, we had to find a methodology that could allow us to access the perception of usefulness. As our sample was relatively small, as only three company's designers were eligible to understand the real significance that describes our hypothesis, we decided to have an inductive [14] research approach inspired by the work of Michelewsky, who demonstrated that one could concentrate on eliciting meaning from empirical data [15]. We choose to have these qualitative research methods to provide learning on particular expectations, experiences, and a person's attitude, problems, needs, ideas, or environment. We carried out an in-depth interview with each designer during February 2021. Each interview had a duration of 45 minutes, was transcribed, and was analysed by identifying the aspects when a project made them feel to understand better the Mexican user and their context in an emphatic way. The quotes had been edited to respect the privacy of the company.

\subsection{Learning about Mexican Culture and the Mexican Family}

These projects aided the designers to understand the Mexican culture, especially on aesthetic values and recognition of how diverse cultures can be as one of the company's designers puts it: "One part that was standing out was that when it came around the form and the colour was very different from Scandinavia." The semantic characteristic of these hypothetical products gave the viewer a more significant dimension on the aesthetic aspects of product design, adding more cultural context than only having a descriptive explanation commonly found on detailed reports. Also, the designers expressed that all projects "accomplished showing some type of insight in relation with the Mexican culture" and felt like "all projects highlighted something and created some type of awareness." The hypothetical products helped the designers to understand the Mexican family on a more empathic dimension. As each product had a specific user with specific needs in a specific context, it helped the designers understand their everyday needs more concretely. Regarding the Negotiation of the Space project, one designer said: "Concrete in the sense that they made it about family life, how that family lives; very tangible by coming up with such a concrete example like: Ok! it is one room, two sisters, they have different needs, and we need to shut the curtain off." Another example of hypothetical products helping the designers to be more emphatic was the project Easy. As they focused on the elderly users, it helped the designers to have a deeper understanding of their frustrations while using newer technology: "That is where I felt getting into the frustration and pain points of the elderly people, like the fear of doing wrong and so."

\subsection{Influence for Designing Future Products}

When asked if these hypothetical products would reach any kind of influence for future projects, the designers mentioned that it helps bring mindfulness of the diversity of the users and the people their company is trying to reach. This could bring "much awareness" into a development process. The designers found these exercises useful not only for a specific project but as a constant learning process where "it helps to read as diverse insights as possible and try to make them stay in the mind of our designers as they execute their work." 
Also, the designers described that the project "Remembrance" caused a change of mind. As product designers tend to look forward when it comes to product design, the characteristic of the project to "look back" into what is familiar for an elder user caused some steer, as a designer explained: "I am quite locked in to thinking that design is only looking forward, is about new things, new expressions... However, how about it also connects back to the past?" To me, that [Remembrance] was an angle which I thought was interesting".

\section{CONCLUSIONS}

The result of this study not only supports the work of Segelström on the usefulness of visual representation to communicate insights, but as these Hypothetical Objects bring an emotional layer of understanding that mere visual representation like a customer journey lack of, as Rosenbaum argues [7]. Turning on our interviews, we asked each designer to complete the sentence "These hypothetical products could___ a user research report." They used the words "enrich," "enable," "enlighten," "complement," and "support", contrasting with what Ross depicts on detailed reports [5]. This study found that the designers felt the user research reports were valuable as they were factual, but the Hypothetical Objects can give a better context. They also expressed that the exercises helped them see distinct aspects away from their surroundings and experiences. For this project, the designers found that they can perceive the colour or flavour on another culture's experience. Also, the designers expressed that the hypothetical projects became "manifestations" of the sum of all the learnings. By looking at them it enabled inspiration, taking a step both for product concepts but also for drawing some conclusions and insights from Mexican culture and how they manifest: "One part that was standing out was that when it came around the form and the colour was very different from Scandinavian."

\section{REFERENCES}

[1] Norman D. A. and Group N. N. (2005). Human-Centred Design Considered Harmful. 5.

[2] Stickdorn M., Hormess M., Lawrence A., and Schneider J. (Eds.). (2018). This is service design doing: Applying service design thinking in the real world; a practitioners` handbook (First edition). O'Reilly.

[3] Netland N., Castanho R., and Sansoucie S. (2012, November 12). UX Strategy on the Job: An Interview with Three UX Strategist. https://www.uxmatters.com/mt/archives/2012/11/uxstrategy-on-the-job-an-interview-with-three-ux-strategists.php.

[4] Holtzblatt K. and Holtzblatt S. (2014). Communicating user research in order to drive design and product decisions. CHI '14 Extended Abstracts on Human Factors in Computing Systems, 11551158. https://doi.org/10.1145/2559206.2559207.

[5] Ross J. (n.d.). Communicating User Research Findings. UX Matters. Retrieved February 8, 2021, from https://www.uxmatters.com/mt/archives/2012/02/communicating-user-researchfindings.php.

[6] Segelström F. (2009). Communicating through Visualizations: Service Designers on Visualizing User Research. 11.

[7] Rosenbaum M. S., Otalora M. L., and Ramírez G. C. (2017). How to create a realistic customer journey map. Business Horizons, 60(1), 143-150. https://doi.org/10.1016/j.bushor.2016.09.010.

[8] Chen R. and Liu J. (2015). Personas: Powerful Tool for Designers. In M. G. Luchs, K. S. Swan, \& A. Griffin (Eds.), Design Thinking (pp. 27-40). John Wiley \& Sons, Inc. https://doi.org/10.1002/9781119154273.ch3.

[9] Chapman C. N. and Milham R. P. (n.d.). The Personas' New Clothes: Methodological and Practical Arguments Against a Popular Method. Th ANNUAL MEETING, 3.

[10] T Harp B. M. and Tharp S. M. (2018). Discursive design: Critical, speculative, and alternative things. The MIT Press.

[11] Malpass M. (2017). Critical design in context: History, theory, and practices. Bloomsbury Academic, an imprint of Bloomsbury Publishing Plc.

[12] Kirby D. (2010). The Future is Now: Diegetic Prototypes and the Role of Popular Films in Generating Real-world Technological Development. Social Studies of Science, 40(1), 41-70. https://doi.org/10.1177/0306312709338325.

[13] Krippendorff K. (n.d.). Product Semantics: Exploring the Symbolic Qualities of Form. 8.

[14] Rainbolt G. W. and Dwyer S. L. (2015). Critical thinking: The art of argument (2e [edition]). 
Cengage Learning.

[15] Michlewski K. (2008). Uncovering Design Attitude: Inside the Culture of Designers. Organization Studies, 29(3), 373-392. https://doi.org/10.1177/0170840607088019. 